\title{
NARRAR Y REÍR EN EL RENACIMIENTO
}

\author{
NUCCIO ORDINE ${ }^{1}$
}

Sería difícil comprender los tratados dedicados en el siglo XVI al tema del relato sin conocer el debate relativo a lo cómico y a la risa que se iba desarrollando en los ámbitos más diversos: de la poética a la filosofía, de la medicina a la fisiognómica. No se trata de cuestiones que conciernen sólo a las teorías estéticas: el análisis de este género literario se refiere también a la fisiología, a la ética y al amplio dominio de las pasiones. El narrar -así como el reír- funciona como un medicamento: ayuda a curar a aquel que escribe y a aquel que lee. Autor y público sacan beneficio del relato cómico.

Antes de estudiar la teoría, será preciso examinar las recopilaciones de relatos y de chistes. El conjunto de tratados, aunque no falten a menudo fines normativos objetivos, acaba siempre por describir unas tendencias y unas prácticas ya muy conocidas. No necesitamos recordar el modelo de Las mil y una noches para entender en general el valor salvador del cuento. Schéhérazade aplaza su propia muerte y cura la locura homicida del rey contando una historia que genera otras historias: la curiositas del verdugo es solicitada de tal forma que el odio hacia las mujeres se transforma en amor.

Sin embargo, es el Decameron, arquetipo por excelencia del género del cuento en Europa, la obra que estrecha los vínculos entre relato y risa. La muerte y la peste se combaten con la ayuda de la laetita («alegría») y del gaudium («gozo»). La vida ordinaria de la alegre brigada en su locus amoenus ( «lugar ameno») se opone al desorden y al caos que reinan en Florencia, ya presa de la epidemia. Boccaccio pinta un cuadro en plena concordancia con el regimen sanitatis ( «régimen de salud») dictado por Tommaso del Garbo ${ }^{2}$, célebre médico florentino de su época:

1 Filósofo y profesor de Literatura Italiana en la Universidad de Calabria. Correo electrónico: nuccio.ordine@unical.it.

2 T. Del Garbo, Consiglio contro a pistolenza, ed. P. Ferrato, Bolonia, Presso Gaetano Romagnoli, p. 40-41 (cap. XXV, «Dell'Allegrezza della mente»). Este texto ha tenido una amplia difusión a lo largo del siglo XV (Florencia, 1522, 1523, 1572) junto con otros opúsculos dedicados a la peste: Modo di conservarsi sano, per regola di vita, non solo quando è la Peste, ma in tutti gl'altri tempi. Tratto da Marsilio Ficino, Tommaso del Garbo, Manardo da Ferrara, Lino da Correggio et altri eccellentissimi Medici, Florencia, Apresso Bartalomeo Sermantelli, 1577; Marsilio Ficino fiorenti Contro alla peste. Insieme con Tommaso del Garbo, Mengo da Faenza, et altri Autori e ricette sopra la medesima materia. Aggiuntovi di nuova una epistola dell'Eccellente Giovanni Mainardi da Ferrara e uno consiglio di Niccolò de' Ranaldi da Sulmona, non più stampati, Florencia, Giunti, 1576 (la Epístola de Manardo da Ferrata, p. 97, fue traducida del latín al vulgar por Niccolò Lorenzini Medico Politiano; es posible, si se acepta la 
Hay que saber que uno de los remedios más perfectos en este caso consiste en alegrarse de manera metódica [...], es decir, en primer lugar, no pensar en la muerte $[\ldots]$ sino llevar los pensamientos hacia cosas agradables y placenteras [...] Y servirse de canciones, juglaría y otros relatos placenteros, que no cansen el cuerpo, y de todas las cosas agradables capaces de aliviar el ánimo³.

A los preceptos relativos a los cuidados corporales les siguen siempre unas indicaciones relativas a los cuidados del alma: evitar pensar en las cosas tristes, intentar divertirse, contar cuentos placenteros e historias capaces de confortar los interlocutores afligidos por preocupaciones graves. El tratado de Del Garbo no es un caso aislado. En toda la literatura médica de la época -hasta finales del siglo XVI- aparece siempre un capítulo o un breve párrafo consagrado a las actividades espirituales necesarias para «conservar la salud y prolongar la vida» ${ }^{4}$. Las enfermedades del alma actúan sobre los cuerpos

hipótesis de un error en el nombre, que se trate deAntonio Lorenzini, más conocido como Poliziano, autor de un tratado sobre la risa: De risu eujusque causis et effectis, Ferrare, 1591). Del Garbo fue amigo de Petrarca y Sacchetti lo menciona varias veces en su Trecentonovelle (XXVI, LXXXVII, CLXVII) ( $c f$. la voz correspondiente cuidada por A. De Ferrari en Dizionario biografico degli Italiani, Rome, 1988, vol. 36, pp. 581585). Sería interesante volver a leer algunos pasajes del Decamerón a la luz de estos preceptos de higiene, sobre todo por lo que concierne al cuidado del cuerpo. En la introducción a la primera jornada y en las diferentes introducciones posteriores, Boccaccio subraya el tema del lavado de manos, sin contar con que, el sábado, las narraciones se interrumpen debido a la exigencia de las mujeres de «lavarsi la testa, di tor via ogni polvere, ogni sudiciume che per la fatica di tutta la passata settimana sopravenuta fosse» (Conclusione della II Giornata: ed. Branca, 2014, p. 316).

3 «E sappi che una delle più perfette cose in questo caso è con ordine prendere allegrezza [...] cioè prima non pensare alla morte [...] ma i pensieri sieno sopra cosa dilettevoli e piacevoli. [...] E usare canzone e giullerie e altre novelle piacevole sanza fativa in corpo e tutte cose dilettevoli che confortino altrui.» La oposición entre risa/tristeza y vida/muerte se dudece claramente de este diálogo entre Dioneo y Pampinea: «[Dioneo] Donne, il vostro senno più che il nostro avvedimento ci ha qui guidati; io non so quello che de' vostri pensieri voi v'intendete di fare: li miei lasciai io dentro dalla porta della città allora che io con voi poco fa me ne usci' fuori. E per ciò o voi a sollazzare e a ridere e a cantare con meco insieme vi disponete (tanto, dico, quanto alla vostra dignità s'appartiene), o voi mi licenziate che io per li miei pensieri mi ritorni e steami nella città tribolata. A cui Pampinea, non d'altra maniera che se similmente tutti i suoi avesse da sé cacciati, lieta rispose: "Dioneo, ottimamente parli: festevolmente viver si vuole, né altra cagione delle tristizie ci ha fatte fuggire"»: Introduzione alla I Giornata (ed. Branca, 2014, p. 42).

4 Castor Durante da Gualdo, Il tesoro della sanità. Nel qual si insegna il modo di conservar la sanità e prolungar la vita e si tratta della natura dei cibi e dei nocumenti loro, (1585), E. Camillo (ed.), Milán, 1982, p. 38. Acerca de estos temas, cf. M. Plaisance, "Funzione e tipologia della cornice», en La novella italiana, Atti del Convegno di Caprarola (19-24 septiembre 1988), Roma, 1989, pp. 103-118. G. Mazzacurati recuerda la función terapéutica del relato en su «Préface» a Conteurs italiens de la Renaissance, Paris, 1993, p. XVIII. 
y las enfermedades del cuerpo actúan sobre el alma. Boccaccio sabe bien que los «relatos placenteros» (piacevoli ragionamenti) alivian los dolores de los enfermos, ayudándoles a curarse, sobre todo cuando la aflicción que les oprime es el mal de amor. Sin embargo, los relatos cómicos no alivian únicamente al autor y a los personajes del Decameron, sino también -por no decir, sobre todo- a los destinatarios de la obra, los cuales acaban por sacar de ello el mayor provecho. El escritor florentino, en efecto, ofrece sus relatos a las mujeres, para ayudarlas a superar la melancolía ${ }^{5}$. Muchos autores volvieron al tema de la melancolía, en sus diferentes acepciones: de Bonaventure des Périers ${ }^{6}$ a Nicolas de Cholières ${ }^{7}$, de Antoine de Saint-Denis ${ }^{8}$ a Philippe d'Alcripe'.

Es suficiente pasar en reseña rápidamente los índices de las colecciones de relatos, los proemios, las epístolas dedicatorias -todos los elementos que Genette denomina umbrales de un texto- ${ }^{10}$ para comprender plenamente la función terapéutica del relato cómico. Función terapéutica que concierne antes que nada a los escritores mismos. Leon Battista Alberti, autor de apólogos y de relatos cómicos, en la epístola dedicatoria de su Musca, dirigida al gran humanista Cristoforo Landino, habla de su experiencia personal: afligido por una fiebre perniciosa, consigue curarse gracias a la risa que le provoca su elogio paradoxal de la mosca: «He escrito la mosca riendo con tanta fuerza

\footnotetext{
5 Proemio (ed. Branca, 2014, pp. 8-9).

6 «J'ay oublié mes tristes passions, / j'ay intermis mes occupations. / Donnons, donnons quelque lieu à folie, // que maugré nous ne vienne saisir, / Et en jour plein de melancholie/ meslons au moins une heure de plaisir»: Bonaventure des Péries, "Les Nouvelles Recreations et Joeyux Devis», (1558), en Conteurs français du XVIe siècle, textos presentados y anotados por Pierre Jourda, París, 1965, p. 365.

7 «Nous sommes battus de la guerre, greslés de l'enjure du ciel, et tellement reduits au petit pied que la tristesse nous accableroit du premier coup, au moins je m'en doute, si nous n'estions relevés par des recreatives consolations propres à nous purger et deschasser de nous la crasse de l'humeur melancholique, qui avec le temps nous perclurroit de notre santé»: Nicolas de Cholières, Les Matinées, en Oeuvres, ed. preparada por Éd. Tricotel, prefacio de Paul Lacroix, París, 1879, t. I, p. 14 (reimpr., Ginebra, 1969)

8 A.D.S.D. [Antoine de Saint-Denis ?], Les Comptes du monde adventureux où sont recitees plusieurs belles Histoires memorables \& propres pour resjouir la compagnie, \& eviter melancholie, París, Par Estienne Groulleau, 1555.

9 «Toutesfois (beaux amis) si au mien conseil voullez assentir, quand sentirez vos espritz estre agravez d'ennuy fantastique et sousy mélancholique, aurez recours au present opuscule, qui divertira chagrin et fascherie en allegresse et joyeuseré [...]»: Philippe D'Alcripe, La nouvelle fabrique des excellents traitcts de verité. Livre pour inciter les resveurs tristes et melancholiques à vivre de plaisir, ed. crítica a cargo de F. Joukovsky, Ginebra, 1983, pp. 11-12.

10 G. Genette, Seuils, París, 1967.
} 
que, a partir de ese momento, la fiebre ha desaparecido a través de un sudor ligero. Me he felicitado conmigo mismo y he agradecido a las moscas que me han hecho recuperar la salud ${ }^{11}$.

Beneficios confirmados por otros autores. Johannes Gast declara haber escrito su Convivialium sermonum liber (1541) para aliviar los dolores insoportables que le causaban unos cólicos nefríticos ${ }^{12}$, mientras que, en el Fuggilozio de Tommaso Costo, "la alegría y la amenidad» (l'allegrezza e la dilettanza) del relato hacen menos insoportables los tormentos de la gota ${ }^{13}$. Nicolas de Cholières atribuye su propia curación a las historias «gallardas y recreativas» narradas durante Nueve mañanas ( Durante nueve mañanas, para engañar mi martirio, he tenido esta hora en la que he podido disfrutar de estos discursos que han hecho desvanecer este aviso de dolor: ipudiera yo transformar el llanto en risa!» $)^{14}$ y Philippe de Vigneuelles declara abiertamente haber escrito Les Cent Nouvelles nouvelles («Los cien nuevos relatos») porque sufría de una "gran enfermedad» ${ }^{15}$. Alberti extiende al público los beneficios del relato cómico. En la dedicatoria de sus Intercoenales, Alberti reivindica, adaptando a sus propias exigencias los versos célebres de Lucrecio, su rol de médico-escritor que hace de la risa un remedio infalible para curar los males del alma:

${ }^{11}$ "Edidi muscam tanto cum cachinno, ut ex ea hora febris tedium, levi sudore evaporato solveretur [...], Congratulor et habeo gratias muscis, quarum ope convalui»: Léon Battista Alberti, «Musca», dans Apologhi ed elogi, ed. a cargo de R. Contarino, Presentación de L. Malerba, Ginebra, 1984, p. 172. Acerca del uso terapéutico de la risa en Alberti, $c f$. las observaciones de Contarino, en particular en su Introduccción (pp. 28-32)

12 "Nos itaque viros imitati clarissimos Amplissime pater a quotidianis molestiis nonnihil liberati et a calculo quo miris cruciatibus ad menses aliquot vexabamur parum quieti veterum ac recentiorum scriptorum iocos et conviviales sermones in unum libellum non citra sudorem concessimus»: Johannes Gast, Convivialium sermonum liber, Basilea, Apud Bartholomeum Westhemerum, 1541, cc. A2v-A3. Los sufrimientos provocados por las piedras en el riñón vuelven en la dedicatoria, dirigida a otro personaje, de la edición publicada en 1543 por la misma imprenta (c. A2).

13 T. Costo, Il Fuggilozio, ed. a cargo de C. Calenda, Roma, 1989, p. 25.

14 Nicolas de Cholières, Les Matinées, cit., p. 8. El autor vuleve varias veces a este tema en la dedicatoria dirigida a Louys de la Chambre: «Or le nombre de neuf qu'icy j'ai retenu, n'est sans mystere: ç'a esté comme une neufvaine, qui m'a guery de mes douleurs, et m'a rendu contant à merveilles. A peine fusmes nous sur la derniere que me voila gay, dispos et sans aucune douleur»: ibid., p. 4

15 " [...] je Phelippe dessusnommez a relevez d'une grande maladie que j'eus en l'an mil cingz cens et cinqz et en maniere de passetemps et attendant santé [...] je me mis lors àa escrire pluseurs asventures [...]»: Philippe de Vigneulles, Les Cent nouvelles nouvelles, ed., intr. y notas a cargo de de Ch.H. Livingston, con la colaboración de F.R. Livingston y R.H. Ivy jr., Ginebra, 1972, p. 57. 
Tú, ciertamente, como el resto de médicos [...] prescribes medicinas tan amargas que provocan náusea. Yo, en cambio, con mis escritos, propongo un modo de aliviar las enfermedades del espíritu mediante la risa y la hilaridad y deseo que resulte claro que lo que pretendo con mis Intercoenales es, sobre todo, que mis lectores se sientan acompañados por hombres divertidos y encuentren en nosotros una ayuda no baladí, para aligerar sus espíritus de las preocupaciones pesadas que los afligen ${ }^{16}$.

El autor sabe también que la cara con la que el médico se presenta al paciente juega un rol determinante en la terapia. Ponerse una máscara cómica o una máscara trágica tal vez pueda resultar determinante. Rabelais -experto en las dos artes: la medicina y la escritura- lo explica claramente en su famosa carta dedicatoria del Cuarto Libro, dirigida a Odet de Coligny:

Existe un pasaje del padre Hipócrates, en el libro que he mencionado, que nos hace sudar, disputar e investigar, para decidir si, en un médico, un rostro triste, tétrico, hosco, catoniano, desagradable, descontento, severo e indescifrable contrista al enfermo, mientras que uno alegre, sereno, gracioso, abierto y agradable lo anima. Esto es algo comprobado ${ }^{17}$.

Las historias que hacen reír, por tanto, constituyen un excelente remedio contra cualquier género de aflicción. Franco Sacchetti, en el proemio de sus Trecentonovelle (¿1385?), habla de atrocidades provocadas por las guerras civiles y de las epidemias de peste ${ }^{18}$. Los males de la guerra y los sufrimientos

16 «Tu quidem ut ceteri physici [...] amaras, et quae usque nauseam moveant, aegrotis corporibus medicinis exhibes; ego vero his meis scriptis genus levandi morbos animi affero, quod per risum atque hilaritatem suscipiatur, ac meis quidem omnibus Intercoenalibus id potissimum a me videri quaesitum cupio, ut qui legerint nos cum facetos fuisse sentiant, cum sibi ad graves curas animi levandas argumenta apud nos non inepta inveniant»: Léon Battista Alberti, «Ad Paulum Toscanellum florentinum», en Opera inedita et pauca separatim impressa, ed. a cargo de G. Mancini, Florencia, 1890 , p. 122.

17 François Rabelais, Le Quart livre, en Les Cinq Livres, ed. a cargo de J. Céard, G. Defaux y M. Simonin, París, 1994, p. 875, para un análisis del rire médical en Rabelais y, más en general, de su concepción filosófica de la medicina, $c f$. R. Antonioli, Rabelais et la médecine, Ginebra, Droz, 1976, (especialmente, pp. 365-364). Indicaciones acerca de la relación entre risa y medicina se encuentran también en M. Lazard, "La Thérapeutique par le rire dans la médecine du XVIe siècle», en Littérature et Patholoque, M. Milner (ed.), París, 1989, pp. 13-27. Acerca de la risa terapéutica, $c f$. también J.-P. Klein, "Rire symptomatique, rire thérapeutique», en La Dérision, le rire, número especial de la revista Internationale de l'imaginaire, n. s, nº 3 (1995), p. 17-25.

18 F. Sacchetti, Il Trecentonovelle, éd. A. Lanza, Florence, 1990, 2e ed., p. 1. 
llevan también a Bonaventure des Périers ${ }^{19} \mathrm{y}$ a Nicolas de Cholières ${ }^{20}$ a componer sus colecciones de relatos. Poggio Bracciolini ${ }^{21}$, Jacques Tahureau ${ }^{22}$, Bénigne Poissenot ${ }^{23}$, Gabriel Chappuys ${ }^{24}$, Ludovico Domenichi ${ }^{25}$, Ludovico Carbone $^{26}$ y Guillaume Bouchet ${ }^{27}$ atribuyen a sus amenidades y «propósitos

19 «Les personnes tristes et angoissées s'y pourront aussi heureusement recréer, et tuer aisement leurs ennuys. [...] De faire à notre age offre de chose tant gentille, je l'ay estimé convenable, mesmement en ces jours tant calamiteux et troublez» Bonaventure des Périers, Les Nouvelles Recreations et Joyeux Devis, («L'imprimeur au lecteur»), cit., p. 363.

20 «Les plaisirs et recreations philosophiques qu'icy j'ay proposé vous serviront d'opiate et asseuré preservatif contre l'air dangereux de ceste saison empestée de tant de malheurs, d'ennuis, de fascheries et de troubles»: Nicolas de Cholières, Les Matinées, (1585), cit., p. 6

21 "Honestum est enim ac ferme necessarium, certe quod sapientes laudarunt, mentem nostram variis cogitationibus ac molestiis oppressam, recreari quandoque a continuis curis, et eam aliquo iocandi genere ad hilaritatem remissionemque converti»: P. Bracciolini, Facezie, con un ensayo de E. Garin, introd., trad. y notass de M. Ciccuto, Milán, 1983, pp. 108-109.

22 «[...] et n'aiant cet œuvre entrepris à autre fin que pour adoucir le travail et recreer le loisir des hommes de sain et entier jugement»: Jacques Tahureau, Les dialogues. Non moins profitables que facétieux, ed. a cargo de M. Gauna, París-Ginebra, 1981, p. 14.

${ }_{23}$ «Je ne passerai plus outre à la commemoration de chose si tedieuse et à toi peu agreable, pour t'adverir que me trouvant de loisir, et privé du confort qu'un homme de lettres peut recevoir par la lecture des livres [...] pour soulager mon esprit aggravé du pesant faix qui le chargeoit, [...] je pensai en moi mesme que, m'occupant en quel que matière joyeuse, les facheries et ennuys qui sans cesse m'assiegeoient, à la venue de leur contraire se dissiperoient et le lairroient en paix»: Bénigne Poissenot (1583), L'Esté, ), ed. a cargo de G.A. Pérouse y M. Simonin, Ginebra, 1987, p. 52.

24 «[...] ayant mes plus serieuses estudes assemblé pour le plaisir et recreation cent facetieuses nouvelles, et considerant que votre tresnoble esprit ne peut estre tousiours bandé et apliqué aux affaires d'importance, [...] je vous le ay hardiment dediees, m'asseurant que [...] vous les accepterez volontieurs et les aurez pour agreables pour y desennuyer vostre esprit genereux et le descharger aucune fois du pesant faix et fardeaxu de voz grands et honorables negoces»: Gabriel Chappuys, Les Facetieuses Journees, París, Jean Houze, 1584.

25 L. Domenichi, Facetie et motti arguti di alcuni eccellentissimi ingegni et nobilissimi signori, Florencia, Torrentino, 1548. Domenichi, además de incluir en su colección una gran parte de los textos de Gast, no duda en adueñarse también de algunos pasajes de la dedicatoria: $c f$. A. Fontes-Baratto, «Pouvoir(s du) rire. Théorie et pratique des facéties aux XVe et XVIe siècles : des facéties humanistes aux trois recueils de Lodovico Domenichi», en Réécritures 3. Commentaires, parodies, variations dans la littérature italienne de la Renaissance, París, 1987, pp. 47-48.

26 L. Carbone, Facezie, cit., pp. 3-4.

27 Guillaume Bouchet, Les Sérées, (1608), con notas e índice a cargo de C.E. Roybet, París, Alphonse Lemerre, 1873, t. I, p. V (reimpr., Ginebra, 1969). En su « 
recreativos» el fin de reanimar las almas cansadas por los continuos negotia («negocios»). Firenzuola, a su vez, escribe unos «relatos divertidos» para consolar un grupo de mujeres entristecidas por la muerte de una amiga común ${ }^{28}$.

El cuidado del cuerpo no puede no tener en cuenta el cuidado del alma ${ }^{29}$. Una confirmación suplementaria podría llegar de un análisis de la ambientación de los relatos. ¿Podemos considerar una casualidad el hecho de que numerosos autores ambientan sus cuentos -o sus colecciones de cuentos-en célebres balnearios? El topos literario del locus amoenus jugará, sin duda alguna, un rol importante a la hora de justificar la ocasión de nobles reencuentros entre personajes de cierto nivel social ${ }^{30}$. Sin embargo, no deben olvidarse tampoco otras posibles influencias en las elecciones de los autores: pensemos en cuadros dominados por situaciones trágicas (epidemias, guerras o desastres naturales) o felices (fiestas, carnavales o una celebración cualquiera).

Aun renunciando a sobrecargar de sentido dichas elecciones, resulta difícil, en cualquier caso, excluir una relación simbólica entre la naturaleza benéfica del relato y la naturaleza benéfica de los balnearios ${ }^{31}$. Lo que cuenta, naturalmente, no es la capacidad real de los relatos de provocar la risa: muchas de estas colecciones, en efecto, no pueden considerarse bajo ningún concepto cómicas. Sin embargo, siguen siendo interesantes -desde nuestro punto de vista- la intención de estos autores y sus constantes alusiones a los efectos terapéuticos de la risa y del relato.

Sabatino degli Arienti titula su colección Porretane, imaginando que unos personajes influyentes de la corte boloñesa de los Bentivoglio se reúnen en el balneario de Porretta para beber la «miraculosa aqua» y contar unos cuentos $^{32}$. El Heptaméron de Margarita de Navarra empieza con la descripción

Discours de l'autheur ", Bouchet considera sus Sérées como un banquete capaz de provocar «l'allegement du corps et recreation de l'esprit» (ibid.)

28 A. Firenzuola, Le Novelle, ed. a cargo de E. Ragni, 1971, p. 16.

29 Acerca de las relaciones entre cuidado del alma y cuidado del cuerpo en el mundo clásico, la obra fundamental es J. Pigeaud, La Maladie de l'âme. Étude sur la relation de l'âme et du corps dans la tradition médico-philosophique antique, Paris, 1981. Cf. también la Introducción de J. Starobinski a Galien, L'Âme et ses passions, París, 1995, pp. VII-XXVI.

30 Cf. M. Minutelli, La miraculosa acqua. Lettura delle Porretane novelle, Florencia, 1990. El autor, además de ofrecer una serie de fuentes relativas a las descripciones literarias de las termas, subraya el aspecto terapéutico de los relatos (especialmente, pp. 31-32).

31 No es casual que un opúsculo dedicado a la risa, escrito en forma de diálogo, tenga como marco «les Bains de Padoue»: G. Ferrari, Democrito et Eraclito. Dialoghi del Riso, delle Lagrime e della Malinconia, Mantua, Appresso Aurelio e Ludovico Osanna, 1627 , p. 1.

32 Sabadino degli Arienti, Le Porretane, ed. a cargo de B. Basile, Roma, 1981, p. 4. 
de los efectos terapéuticos del balneario de Caudères (Cauterets), en los Pirineos: «Unos van para beber agua, otros para bañarse y oros para disfrutar de los barros, todas cosas tan maravillosas que los enfermos vuelven a casa completamente curados» ${ }^{33}$.

Sermini, a su vez, envía sus relatos a su amigo que, al encontrarse en el balneario de Petriolo, cerca de Siena, le pide un ejemplar después de haberles oído a unos amigos comunes hablar de ellos ${ }^{34}$. Matteo Bandello, en una epístola dedicatoria dirigida a Girolamo Fracastoro, describe el balneario de Caldiero, cerca de Verona, como un lugar en el cual, durante los momentos de descanso, se cuentan unos cuentos ${ }^{35}$. Y Scipione Bargagli, en sus Trattenimenti, inventa el gioco del bagno («juego del baño») para aliviar el mal de amor ${ }^{36}$.

Las dos terapias -las aguas termales, por un lado, y los relatos divertidos, por el otro- procuran un alivio profundo del cuerpo y del alma. Además, favorecen los más variados procesos «liberatorios». El testimonio que nos ofrece Floriano Dolfo -en una carta cómica dirigida al marqués de Gonzaga- pone en evidencia la manera en la que los balnearios pueden revelarse «liberatorios», no sólo desde un punto de vista fisiológico:

Los que frecuentan los baños carecen de cualquier reverencia y pudor: no les da vergüenza tirarse pedos, eructar, hacer caca y pis en público, enseñar a menudo y sin enrojecer sus culos, sus penes y sus vaginas, algunas rotas, sangrantes y sucias, y culos llenos de crestas y almorranas, negros, rojos o blancos, y penes desmedidos, contra los cuales no podría competir ni un burro. Y hombres y mujeres van a los baños juntos, entran desnudos en el agua y allí, con los pies y con las manos, y con palabritas de amor, disfrutan de mucho placer ${ }^{37}$.

33 Marguerite de Navarre, L'Heptaméron, ed. a cargo de M. François, París, 1991, p. 1.

34 G. Sermini, Novelle, Livourne, Francesco Vigo, 1874, p. 3.

35 M. Bandello, La seconda parte delle novelle (II, 9), en Tutte le opere, ed. F. Flora, Milán, 1966, 4e ed., vol. I, pp. 726-727. Aunque se trata de un cuento trágico, la descripción de los baños de Caldiero como un lugar en el cual, en los momentos de pausa, se cuentan relatos es importante. Bandello, por otra parte, había tratado ya este tema en la dedicatoria dirigida a Sforza Bentivoglio (I, 21). Jérôme Fracastor intervino con fuerza en el debate acerca de la teoría de la risa, con el capítulo «De admiratione et ecstasi et risu ", en su De Sympathia et anthipathia rerum, Lugduni, Apud Ioan. Tornaesium, 1554 (una edición anterior había sido publicada en Venecia en 1546).

36 S. Bargagli, I Trattenimenti, ed. L. Riccò, Roma, 1989, pp. 498-530.

37 «Ogni reverentia et pudore è alieno da li bagnaroli, che non si vergognano petare, cacare, rutare et pissare in pubblico, mostrando spessisime volte senza rubore li culli, cazi et pote, de le quale si ne vede alcuna fracassata piena di bardelle et tute sanguinate et lerze, et culli pieni di creste et morene, chi negro chi rosso chi bianco, et vèdesse li più smisurati cazi che a comparazione li asinini parebono niente [...]. Et insieme maschi et femine vanno al bagno et entrano ne l'acqua ignudi et qui cum piedi et mano et parolete 
El jesuita Étienne Binet -consciente del riesgo de caer del humor en la obscenidad y en lo ilícito-, en su colección Consuelo, instrucción y alegría para los enfermos y las personas afligidas $(1617)^{38}$, elabora la teoría del «humor piadoso» para provocar la curación de los pacientes mediante la intermediación de relatos protagonizados por mártires y santos ${ }^{39}$. Sin embargo, las precauciones con las que trata lo sagrado y lo profano no le convencen a Pascal, que, en un pasaje de sus Provinciales, fustiga así al pobre Binet:

Empezando por la manera indigna con la que esos vuestros autores hablan de cosas santas, tanto en sus burlas como en sus galanterías y en sus discursos serios, ¿creéis que todos esos cuentos ridículos de vuestro Padre Binet, en su Consuelo de los enfermos, son de verdad apropiados a su propósito de consolar cristianamente a los que Dios aflige? ${ }^{40}$

En efecto, el vínculo entre la risa y el relato pasa también por la vis comica y el eros. Poggio Bracciolini vuelve muy a menudo sobre este tema, mostrando en una de sus bromas como una buena dosis de sexo puede, al mismo tiempo, curar la locura en las mujeres y desencadenar la hilaridad en los pacientes frenéticos y entre los testigos estupefactos. La historia merece ser contada:

Una mujer de mi aldea, que todos consideraban frenética, era llevada por su marido y sus parientes a casa de otra mujer, de la que se decía que era bruja y con la que todos contaban mucho para curar a la enferma. Llegados a un vado del río Arno, pusieron a la mujer sobre las espaldas del hombre más robusto. Enseguida, hete aquí que ella empieza a mover sus nalgas como si estuviera copulando y a gritar varias veces con todas sus fuerzas: «¡Quiero que me jodan!». Así, ella daba

amorose se pigliano grandissimo piacere»: cit. en P. Stoppelli, «Facezie oscene inedite di Floriano Dolfo a Francesco I Gonzaga », Belfagor, XXXII, 1977, p. 691.

38 E. Binet, Consolation et Réjouissance, texto presentado y anotado por C.L. Combet, Grenoble 1995 (cf. Recueil des Euvres spirituelles du P. Estienne Binet, Rouen, 1627).

39 Cf., p.ej., las ironías de Binet acerca del martirio de los santos : «Le Paradis est la salle des noces de l'Agneau, ici-bas on fait l'appareil et la cuisine: ô le beau ! ô le rare ! et ô le superbe service !Chaque saint y porte son plat en très belle ordonnance. Saint Étienne présente en un plat d'or des chairs hachées à coups de pierres ; saint Jean, des autres frites à l'huile ; saint Laurent, des membres rôtis sur le gril ; saint Vincent, des viandes salées [...]» : ibid., p. 9. Para un retrato de Binet, $c f$. la «Préface» de M. Fumaroli a É. Binet, Essay des merveilles de nature, et des plus nobles artifices, Évreux, Association du théâtre de la ville d'Évreux, 1987, p. 9-50 (y, también, de Fumaroli, L'École du silence. Le Sentiment des images au XVIIe siècle, París, 1994, ad indicem).

40 Blaise Pascal, «Onzième lettre écrite par l'auteur des Lettres au provincial aux Révérends Pères Jésuites », en Les Provinciales, ed. a cargo de M. Le Guern, Paris, 1987. P. 182. Acerca de la risa en Pascal, $c f$. J. Morel, "Pascal et la doctrine du rire grave», en Méthodes chez Pascal, París, 1979, pp. 213-219. 
a conocer su enfermedad. El que la llevaba fue presa de una risa tan fuerte que se cayó en el agua junto con la mujer. Los demás también soltaron una carcajada cuando comprendieron de qué mal sufría la mujer y cuál era el remedio necesario para su curación ${ }^{41}$.

El gesto, obsceno en sí, provoca la risa y la risa provoca la curación. En el Cunto de li cunti ( Cuento de los cuentos») de Gianbattista Basile, la princesa Zoza no se ríe nunca, porque sufre de melancolía. Un día, desde una ventana del palacio, la joven asiste a una escena que se desarrolla en la calle: una vieja enfadadísima con un joven paje se levanta el vestido y enseña sus partes y, aunque ella no sea la mítica Baubo, su gesto atrevido obtiene el mismo resultado, haciendo estallar una risa benéfica en los labios de la princesa afligida ${ }^{42}$.

La lista de curaciones no remite únicamente a comportamientos obscenos. En muchos relatos se encuentran ejemplos de enfermos, incluso de enfermos próximos a la muerte, que recuperan la salud después de un estallido de risa imprevisto. Pontano -teórico del humor- cuenta la historia de un pobre cura que, después de haber probado sin éxito diversos remedios

41 «Mulier ex meo municipio, cum videtur phrenetica, ducebatur a viro et genere proximis ad foeminam faticam quondam, cuius ope vel opere curaretur. Cum per Arnum fluvium transituri mulierem supra dorsum hominis validioris imposuissent, coepit illa e vestigio nates movere, similis coeunti, ac magna voce clamitans, "Ego" inquit saepius verba iterans "vellem futuiri" quibus vocibus causam expressit morbi. Qui ferebat foeminam, adeo est in risum effusus, ut una cum ea in aquam caderet. Tu ridentes omnes, cum insaniae medelam cognovissent, non esse opus incantationibus asserunt, sed coitu, ad sanitatem restituendam» Poggio Bracciolini, Facezie, cit., p. 144-146.

42 G. Basile, Lo cunto de li cunti, ed. a cargo de M. Rak, Milán, 1986, pp. 10-12. Poco después, Basile, por mediación de Taddeo, subraya el poder terapéutico del relato, que consiste en su capacidad de aliviar los dolores del parto: "Non è chiù cosa goliosa a lo munno, magne femmine meie, quanto lo sentire li fatti d'autro, né senza ragione veduta chillo gran felosofo mese l'utema felicità de l'ommo in sentire cunte piacevole, pocca ausolianno cose de gusto se spapurano l'affanne, se da sfratto a li penziere fastidiuse e s'allonga la vita [...]. Per la quale cosa devo scusare moglierema, se l'è schifaffato 'n capo sto omore malanconeco de sentire cunte. Però se ve piace de dare ' $m$ brocca a lo sfiolo de la precepessa mia e cogliere 'miezo a le voglie meie, sarrite contente, pe sti quattro o cinque iurne che starà a scarrecare la panza, de contare ogni iornata no cunto ped uno, de chille appunto che soleno dire le vecchie pe trattenimento de peccerille»: ibid., p. 22. Acerca del rol de la risa en este texto, además de la introducción de Rak (pp. 1059-1063), cf. P. Guaragnella, Le maschere di Demcrito e di Eraclito. Scritture e malinconie tra Cinque e Seicento, Fasano, 1990 (especialmente los caps. III y IV). (Cf. también S. Nigro, «Popolo e popolarità», dans Letteratura Italiana Einaudi. Le Questioni 5, Turín, 1986, p. 231). Acerca del mito de Baubo que hace reír a Deméter enseñándole sus genitales, $c f$. G. Devereux, Baubo. La vulve mythique, París, 1983. 
para aliviar unos dolores intestinales atroces, es salvado, sin darse cuenta, por una carcajada. Un chiste contado sin preaviso por un criado produce una providencial reacción en cadena: la risa provoca el pedo que permite al enfermo liberar el vientre hinchado ${ }^{43}$.

Acciones cómicas protagonizadas por monos ocupan un lugar destacado en la lista de efectos terapéuticos de la risa. Cristofaro Armeno cuenta cómo un rey afligido por la melancolía por haber ordenado la muerte de su propia mujer recupera alegría y salud gracias a los juegos de un mono ${ }^{44}$. Heinrich Bebel pone en escena a un médico enfermo a punto de morir: mientras sus criados se adueñan de sus bienes, el mono entra tranquilamente en la habitación y se pone en la cabeza el birrete de su dueño: ... unde dominus in risum est affusus atque convaluit («y por esto el señor se echó a reír y se curó») ${ }^{45}$. Bonaventure de Périers reescribe el relato cambiando algunos elementos: el enfermo en la cama ve un mono que bebe su medicina:

En fin, el mono tanto hizo, tocando aquí y allá, que acabó con encontrar esta medicina y con bebérsela hasta el fondo para luego lamerse sus barbas. Mientras tanto, el enfermo, que lo estaba mirando, probó un placer tan grande al ver las caras que ponía el animal que se olvidó de su mal y empezó a reírse con tanta fuerza y con tantas ganas que se curó completamente. Porque, gracias a una alegría repentina e inesperada, los espíritus recuperan sus fuerzas, la sangre se rectifica y los humores vuelven a su sitio, tanto es así que la fiebre desaparece ${ }^{46}$.

Si los autores de relatos y bromas emplean unos tratados de medicina, los médicos, a su vez, se sirven de la literatura para mostrar unos ejemplos vivaces capaces de apoyar sus hipótesis. Laurent Joubert, médico y filósofo

43 G. Pontano, Antonius, en I dialoghi, ed. a cargo de G. Previtera, Florencia, 1943, p. 55. No hay que olvidar la importancia del De Sermone de Pontano en la teoría de la facetudo: $c f$. G. Ferroni, "La teoria classica delle "facezie" da Pontano a Castiglione», Sigma, XIII (1980), pp. 69-96.

44 Cristofaro Armeno, Peregrinaggio di tre giovani figliuoli del re di Serendippo, dans Novelle del Cinquecento, ed. a cargo de G. Salinari, Turín, 1976, pp. 380-381. Esta colección (Venise 1577) -en la que puede leerse el relato en el que aparece el mono (La regina che per prima riuscì a far scolpire il suo nome sulle monete)- è presentada por el autor como una traducción italiana de relatos persas.

45 H. Bebel, Facetien, drei Bucher, ed. a cargo de G. Bebermeyer, Leipzig, 1931, p. 119 (III, 38 «De Simia»). Acerca de la difusión de Bebel en Francia, $c f$. M. Simonin, "Bebel en France au XVIe siècle: le cas des "Comptes du monde adventureux" ", Journal of Medieval and Renaissance Studies, XIII (1983), pp. 269-291. Se refiere sin duda a este relato Stefano Guazzo, La civil conversazione, ed. a cargo de A. Quondam, Módena, Panini, 1993, pp. 295-296.

46 B. Des Périers, Nouvelle Récréations et Joyeux Devis, éd. K. Kasprzyk, París, 1980, pp. 305-304 (n89, «Du Singe qui beut la medecine»). 
influyente, consagra a este sujeto un capítulo de su Tratado sobre la risa que lleva por título «Cuál es el bien que aporta la Risa y si es posible que un enfermo se cure a fuerza de reír», en el que cuenta tres historias en las cuales aparecen un enfermo y un mono ${ }^{47}$. Naturalmente, el primate, nuestro lejano pariente - que Galeno considera uno de los animales más ridículos debido a su capacidad de imitar sin armonía las acciones de los hombres- ${ }^{48}$, ocupa en los tres casos, sin darse cuenta, el lugar del médico:

Así, en estos enfermos, las acciones divertidas de los monos (animales en sí ridículos) excitan y levantan la naturaleza abrumada, abatida y como sofocada del mal. Lo que puede conllevar fácilmente el placer de la risa, porque semejante alegría mueve el calor languidecido y enterrado, lo expande a través del cuerpo entero y lo lleva a ayudar la naturaleza. La dignidad y la excelencia de la Risa, pues, es muy grande porque refuerza el espíritu tanto que ella puede modificar de repente el estado de un enfermo y llevarlo a curarse aun en punto de muerte ${ }^{49}$.

Las terapias, sin embargo, deben elegirse con atención extremada: un remedio tomado en dosis excesiva puede transformarse en instrumento de muerte. De la misma manera, la risa debe ser administrada en las justas proporciones para conseguir unos efectos beneficiosos ${ }^{50}$. Más allá de ciertos

47 L. Joubert, Traité du ris, París, Chez Nicolas Chesneau, 1579, (III, XIV), p. 335 (reimpr., Ginebra, 1973). Su interés por la lengua y la literatura es atestiguado por un diálogo (Dialogue sur la cacographie française) publicado como apéndice al tratado sobre la risa (ibid., p. 376-399). El diálogo es precedido por la traducción francesa de la célebre carta de Hipócrates a Damagetes (ibid., pp. 355-375). Une versión abreviada en latín del Traité $d u$ ris fue tal vez publicada en 1558, aunque tres ediciones $(1560,1567,1574)$, todas impresas en Lyon, preceden la de 1579 (Cf. P.-J. Amoreux, Notice historique et bibliographique sur la vie et les ouvrages de Laurent Jobert, Montpellier, J.G. Tournel, 1814, p. 26 ; reimpr., Gonebra, Slatkine, 1971). La historia del mono narrada por Joubert será retomada en 1582 en un tratado de Paré: A. Paré, Euvres complètes, t. I, ed. a cargo de J.F. Malgaigne, París, Imprimerie de Bourgogne, 1840, p. 95.

48 Galien, L'Utilité des parties du corps humain (II, XXII). Se encuentra también una alusión al mono en los Procédés anatomiques, IV-1, 415. Hombre y mono se encuentran asociados también en el Liber medicinalis de Quinto Sereno Sammonico (cap. XLIV: Hominis vel simiae morsu: «Sive homo seu similis turpissima bestia nobis/ vulnera dente dedit, simul intulit atrum [...]»): cf. Quinto Sereno Sammonico, Libert medicinalis, ed. a cargo de C. Ruffato, Turín, 1996, p. 98.

49 L. Joubert, Traité du ris, cit., p. 335.

50 En una facezia de Sozzini, un personaje enferma por el mucho reír: A. Sozzini, Raccolte di burle, facetie, motti e buffonerie di tre huomini sanesi, Sienne, Onorato, 1865, p. 60; reimpr., Bolonia, 1975. 
límites, en efecto, el tratamiento puede producir reacciones completamente opuestas. La noción de pharmakon evoca, por otra parte, un medio capaz a la vez de poner fin a la enfermedad o a la vida ${ }^{51}$. Joubert, en el capítulo XV, que lleva por título «Cuál es el mal que provoca la Risa pródiga y demasiado continua», explica que "no hay nada tan útil y tan agradable que no pueda volverse dañino y triste, si se usa de forma demasiado seguida ${ }^{52}$. El tema es tan delicado que, en las últimas páginas del tratado, se pasan en reseña unos casos en los que la muerte se debe a exceso de risa ${ }^{53}$. Bien entendido, Joubert, en su análisis, se apoya en un repertorio de exempla que, partiendo de

51 Acerca de la doble conotación del vocablo pharmakon, en relación con el Fedro de Platón, cf. J. Derrida, «La pharmacie de Platon», en La Dissemination, París, 1972, pp. 69-196.

52 L. Joubert, Traité du ris, cit., p. 336.

53 Joubert dedica a este sujeto («Savoir si quelqu'un peut mourir de rire») un capítulo especial (III, XVI, pp. 345-352). Numerosos autores mencionan los efectos negativos y mortales de la risa: I. C. Scaligeri, «Risus», en De subtilitate (Exercitatio CCCXVIII, par. 6), Lutetiae, Apud Federicum Morellum, 1557, c. 431v (Scaligeri se ocupa también de lo cómico en su Liber de comicis dimensionibus, Apud Seb. Gryphium, 1539); R. Goclenii, Physiologiae Risus et Ridiculi, Marpurgi, Paulus Egenolphus, 1597, p. 26; Elpidio Berrettari, Tractatus de Risu, Florentiae, Apud Cosmum Iuntam, 1603, c. 26v; N. Nancelii, De Risu libellus, en De immortalitate animae, Parisii, Apud Ioannem Richerium, 1587, c. 87v; H. Gutberleth, «Laetitiae accidunt Gratulatio et Risus», en Pathologia hoc est Doctrina Humanis affectibus, Hebornae Nassaviorum, 1615, p. 25; G. Ferrari, Democrito et Eraclito..., cit., pp. 14, 38, 40 (cf. también pp. 31-32); Anónimo, Des passions humaines de la joie et de la tristesse et quelle est la plus véhémente, en Édouard Fremy, L'Académie des derniers Valois, París, Ernest Leroux, 1887, p. 249. Acerca de la expresión «morir de risa» en el mundo clásico, $c f$. D. Arnould, Le Rire et les Larmes dans la littérature grecque d'Homère à Platon, París, 1990, pp 222-223 (pero $c f$. también L. Callebat, «Le rire et la mort. Effets d'exclusion et dramaturgie», en La Mort, les Morts et l'Au-delà dans le monde roumain, ed. a cargo de F. Hinard, Caen, 1987, pp. 257-262). Alude a este tema también C. Weyman, «Zu den Sprichwörtern und sprichwörtlichen Redensarten der Römer», en Archiv für Lateinische Lexicographie und Grammatik, 8 (1893), pp. 408-409. La literatura italiana relativa a este sujeto es estudiada ampliamente por E. Scarpa, «Morire dal ridere» en Lingua nostra, XXXVIII (1977), pp. 101-107. Acerca de los personajes muertos de risa en la literatura del siglo XVI, cf. S. Longhi (Lusus, Il capitolo burlesco nel Cinquecento, Padua, 1983) y G. Ferroni («Morti dal ridere e carnevali letterari», en Il Carnevale: dalla tradizione Arcaica alla traduzione colta del Rinascimento, Atti del XIII convegno del Centro Studi del Teatro Medievale e Rinascimentale, Roma, 31 maggio-4 giugno 1989, pp. 391-408). Puede encontrarse un amplio repertorio del uso terapéutico de la risa en los relatos italianos en J. Lacroix, "Esquisse d'une signification du rire chez les nouvellistes italiennes des XIIIe, XIVe et XVe siècles», en Le Rire au Moyen Âge dans la littérature et dans les arts, Actes du colloque international des 17-19 novembre 1988, Bordeaux, 1990, pp. 201-225. 
Valerio Máximo ${ }^{54}$, llegan, a través de numerosos añadidos, hasta Rabelais ${ }^{55}$, Jean Tixier de Ravisy ${ }^{56}$ y Ortensio Lando ${ }^{57}$.

Los relatos podrían provocar también, por diferentes razones, unos efectos mortales. Un cuento mal contado, por ejemplo, se vuelve una amenaza para la vida de los destinatarios. Bocaccio ofrece un ejemplo de esto en el relato que abre la sexta jornada de su Decameron, consagrada a los chistes y a los juegos de palabras. En el centro del Decameron, como narración enmarcada en las otras, el autor pone en escena las peripecias de un caballero naif que se improvisa cuentacuentos: la repetición, el hecho de corregirse constantemente y de volver al punto de partida, el lenguaje inadecuado e insuficiente producen un efecto devastador en el relato-la cuale nel vero da sé era bellissima ( el cual, en sí, era verdaderamente hermosísimo»)- y en la desventurada oyente: Di che a Madonna Oretta, udendolo, spesse volte veniva un sudore e uno sfinimento di cuore, come se inferma fosse stata per terminare

54 Valère Maxime, «De mortibus non vulgaribus», en Dits et faits mémorables (IX, 12, 6). Cf. también Sextus Pompeius Festus, De Verborum significatu quae supersunt cum Pauli Epitome, ed. W.M. Lyndsay, Leipzig, 1933, p. 228: aquí se narra la célebre anécdota del pintor Zeuxis que muere de risa a la vista de una vieja muy fea representada en uno de sus cuadros.

55 Acerca de las alusiones de Rabelais a los casos de muerte por exceso de risa, $c f$. C. Quesnel, Mourir de rire d'après et avec Rabelais, Montreal-París, 1991 (cf. también N. Ordine, «L'âne comme les Silènes: les apparences sont trompeuses», en Le Mystere de l'âne. Essai sur Giordano Bruno, París, 1993, pp. 105-106). En la literatura del Renacimiento, un ejemplo célebre es el de la muerte de Margutte descrita por Luigi Pulci en su Morgante: Margutte muere viendo un mono que se pone sus botas («e poi che fu della bertuccia accorto / vide ch'egli era per le risa morto»: L. Pulci, Morgante, ed. a cargo de F. Ageno, Milán-Napoles, 1955, XIX, 149, 7-8, p. 592). Este episodio es mencionado en relación con el tema de la muerte como espectáculo por R. Gigliucci, Lo spettacolo della morte. Estetica e ideologia del macabro nella letteratura medievale, Anzio, De Rubeis, 1994, pp. 70-72 (cf. también E. Scarpa, «Un'ipotesi sulla morte di Margutte», en Filologia e critica, I, 1976, pp. 138-144). También Le Tasse, se refiere a la fuente de la risa en la isla de la maga Alcina, fuente capaz de estimular la risa hasta la muerte: "Un fonte sorge in lei che vaghe e monde / ha l'acque sì che i riguardanti asseta; / ma dentro a i freddi suoi cristalli asconde / di tosco estran malvagità secreta, / ch'un picciol sorso di sue lucide onde / inebria l'alma tosto e lo fa lieta, / indi a rider uom move, e tanto il riso / s'avanza alfin ch'ei ne rimane ucciso" (Gerusalemme liberata, XIV-74).

56 Io. Ravisii Textoris Nivermensis Officina partim hustoriis partim poeticis referta disciplinis, París, R. Chauldière, 1520, f. 35.

57 O. Lando, Catalogo di tutti quelli che morirono per soverchia letitia o per smoderate risa, en Sette libri de cathaloghi a varie cose appartenenti non solo antiche, ma anche moderne, Vinegia, Giolito de' Ferrari, 1552, pp. 91-94. Lando, en esta obra (p. 92), hace referencia al tratado sobre la risa de A. Lorenzini, más conocido como Policiano. 
(«Por lo cual a Madonna Oretta, oyéndolo, le entraban a menudo un sudor y un cansancio de corazón, como si estuviera enferma y a punto de fenecer» $)^{58}$.

Se trata de problemas -en los cuales nos hemos centrado brevemente hasta aquí- que no podían ser ignorados por los teóricos del relato. Francesco Bonciani, Girolamo Bargagli y Francesco Sansovino ${ }^{59}$ no escriben verdaderos tratados: sus intervenciones, sin embargo, ocupan su lugar en el gran debate relativo a la codificación y normalización de los géneros literarios que había empezado a desarrollarse en Italia ya a partir de la segunda mitad del siglo XVI. La Lezione sopra il comporre delle novelle («Lección sobre la composición de relatos») de Bonciani (1574), en particular, parece tener un objetivo teórico más destacado. Las reflexiones de los otros dos autores se enmarcan, al contrario, en un contexto más ligero: Bargagli habla del juego del relato en su Dialogo de’ Giuochi («Diálogo de los Juegos», 1572) y Sansovino consagra un párrafo al arte delle novelle ( «arte de los relatos») en su prefacio a la antología Cento novelle scelte («Cien relatos escogidos»), publicada en Venecia en 1571.

En estos textos, el interés se centra exclusivamente en el relato cómico. El relato trágico está expresamente prohibido debido a sus efectos negativos. Es suficiente volver a leer la definición sintética y eficaz ofrecida por Bonciani para comprender el sentido de una elección que podría parecer extraña: diremo che le novelle sieno imitazione d'una intera azione cattiva secondo il ridicolo, di ragionevol grandezza, in prosa, che per la narrazione genera letiza ( «diremos que los relatos son la imitación de una completa acción mala según lo ridículo, de amplitud razonable, en prosa, cuya narración genera alegría» $)^{60}$. Aquí, el acento cae sobre la identificación completa entre relato y risa.

Sansovino y Bargagli se encuentran en la misma línea. Si el primero se limita a repetir que la «fábula» es «empleada comúnmente por las mujeres en todas las Provincias y en todas las Ciudades, en privado y en sus brigadas, para alegrarse y divertirse ${ }^{61}$, el segundo subraya con fuerza su desaprobación de los relatos trágicos: «Yo no apruebo tampoco que se cuenten esos relatos que

58 Boccaccio, Decameron, VI, 1 (ed. a cargo de Branca, 2014, pp. 718-179).

59 Francesco Bonciani, Lezione sopra il comporre delle novelle (1574); Girolamo Bargagli, Dialogo de' i giuochi (1572); Francesco Sansovino, Dell'arte delle novelle, en Cento novelle scelte da più nobili scrittori della lingua volgare con l'aggiunta di cento altre novelle antiche (1571), Los tres tratados fueron publicados juntos por primera vez como apéndice del volumen de N. Ordine, Teoria della novella e teoria del riso nel Cinquecento, Nápoles, 1996, al cual remiten las citas.

60 F. Bonciani, Lezione sopra il comporre delle novelle, cit., p. 111.

61 F. Sansovino, Dell'arte delle novelle, cit., p. 161. 
terminan en la tristeza y en la pena, porque, allí donde uno espera del relato que provoque gozo y alegría, ellos no aportan sino dolor y melancolía ${ }^{62}$.

El relato, en efecto, debe imitar un'azione cattiva secondo il ridicolo ("una acción mala según lo ridículo»), con el fin preciso de generar en los destinatarios «alegría» y placer. Bonciani, Bargagli y Sansovino subrayan en numerosas ocasiones la necesidad de que el relato favorezca el reposo y, al mismo tiempo, ayude a los hombres a sobrellevar las tristezas de la vida. Tras esta idea no es difícil ver ciertos pasajes de la Ética a Nicómaco en los que se hace alusión a la eutrapelia, es decir a la capacidad de divertirse alegremente manteniendo siempre un comportamiento digno y alejado de cualquier exuberancia excesiva ${ }^{63}$. La risa moderada podrá también "purgar» el alma de los dolores que nos afligen.

Ahora bien, ¿cómo provocar la risa? La cuestión, debido a las razones que hemos indicado, juega un rol importante en la teoría del relato. Bonciani afirma que la "maravilla» (admiratio), en el sentido de "estupor», es una de las causas principales de la risa. El lector necesita encontrarse cara a cara con gestos o acciones inesperados que impliquen necesariamente la novità (la «novedad», lo «inusitado»). El autor debe, en resumidas cuentas, construir una situación imprevista, rara, inusitada. El vínculo entre diletto («placer») y novità («novità») es también subrayado por Bargagli. El relato que quiere aportar «placer [...] a los oyentes» debe, en efecto, «tener algo nuevo y remarcable e implicar cierta verosimilitud en su extrañeza ${ }^{64}$. En las páginas de Bonciani y de Bargagli, volvemos a encontrar los mismos temas, objeto de debates en el conjunto de tratados dedicados a la risa: de Valleriola a Antonio Lorenzini, de Berrettari a Goclenio, de Paleotti a Francisco de Valles ${ }^{65}$. Fracastor, en particular, en un capítulo de su De sympathia et antipathia rerum («Sobre la simpatía y la antipatía de las cosas») que lleva por título «De admirationi, et ecstasi et risu» («De la admiración y del éxtasis y de la risa»), estudia con atención el vínculo que une lo «nuevo»y la «maravilla»:

Lo que provoca específicamente la risa son las cosas nuevas, inusitadas, improvisas, ligeras y divertidas, que contienen alguna alusión sutil a otras cosas. [...]

62 G. Bargagli, Dialogo de'i giuochi, cit., p. 146.

63 Aristotele, Etica Nicomachea, IV, 14, 1128 a 4-16, Acerca de la eutrapelia, $c f$. también II, 7, 1108 a 23-26. Acerca de la recuperación del concepto de eutrapelia en el Renacimiento, en particular en Erasmo y Noël du Fail, $c f$. D. Ménager, La Renaissance et le rire, París, 1995, pp. 86-89.

64 G. Bargagli, Dialogo de'i giuochi, cit., p. 219.

65 Acerca de las diferentes posiciones de estos autores, $c f$. Nuccio Ordine, Teoria della novella..., cit., pp. 59-91. 
Todas estas cosas, en efecto, si se presentan de manera novedosa, precisamente en virtud de su novedad, producen una admiratio («maravilla») inmediata ${ }^{66}$.

Vincenzo Maggi, en su De ridiculis (1550) -partiendo de unas afirmaciones de Aristóteles contenidas en su Retórica «El aprender y el maravillarse son en la mayoría de los casos agradables: de un lado, maravillarse implica desear (aprender); por consiguiente, el maravillarse es deseable») ${ }^{67}$ - analiza la relación res novaeladmiratio ( «cosas nuevas/maravilla») a la luz de los mecanismos que presiden el conocimiento y el placer que de ellos deriva. Maravillarse y conocer, en efecto, implican necesariamente la novedad: lo nuevo es el producto de la "mutación» (de un cambio) así como el acto de conocer presupone el deseo de aprender lo desconocido y, por tanto, lo «nuevo». Laurent Joubert va todavía más allá. Él abre su Traité du ris («Tratado sobre la risa») con la evocación de un mito tomado de la Teogonía de Esíodo, que atribuye a Thaumas la paternidad de Iris:

Esíodo, autor de fabulosas invenciones y filósofo divino, queriendo significar que la maravilla que se prueba ante los efectos de la naturaleza engendra preguntas y, finalmente, conocimiento de las causas, ha imaginado ingeniosamente que Irisi era la hija de Thaumas para demonstrar que los que no fueran presa de un estupor y de un curioso deseo, no se harán jamás preguntas y, por consiguiente, no encontrarán ninguna respuesta ${ }^{68}$.

La contemplación (Iris), por tanto, es generada por la maravilla (Thaumas). Esta imagen simbólica había sido desarrollada ya en el Teeteto de Platón («Porque este sentimiento, el maravillarse, es propio de alguien que ama el saber. No hay otro punto de partida para la búsqueda del saber que aquél y quien dijo que Iris nació de Thaumas no ha construido mal la genealogía» ${ }^{69}$ ), diálogo en el cual Sócrates atribuye explícitamente a la admiratio la generación de la filosofía misma ${ }^{70}$.

66 «Res autem quae proprie risum movent, sunt in primis novae, repentinae, leves et lidicrae, habentes argutiam quandam et concinnitatem ad aliud [...]. Haec enim omnia uti nova offerentur et propter novitatem subitam admirationem inducunt»: Fracastor, De sympathia et antipathia rerum, cit., p. 119.

67 Aristóteles, Retórica, I, 11, 1371 a 25-33.

68 L. Joubert, Traité du ris, cit., p. 1. Este mito es recuperado por Montaigne: «Iris est fille de Thaumantis. L'admiration est fondement de toute philosophie, l'inquisition le progrez, l'ignorance le bout» (Montaigne, Les Essays, III, 11, ed. a cargo de P. Villey, París, 1988, p. 1030). Cf. D. Ménager, La Renaissance et le Rire, cit., pp. 38-39.

69 Platón, Teeteto, 155 d.

70 La misma idea en Aristóteles (Metafísica A 2, 982 b 12-21). Patrizi se fija también en el vínculo entre admiratio e ignorancia: F. Patrizi da Cherso, Della Poetica, cit., vol, II, p. 368. El placer de la búsqueda de las novedades es subrayado también 
Sin embargo, la relación entre la admiratio y la ignorancia implica una serie de consecuencias. En primer lugar, puede ser útil para explicar un problema que hunde sus raíces en el mundo clásico: ¿por qué los sabios ríen raramente o no ríen nunca ${ }^{71}$ En efecto, se trata de comprender cuáles son las causas que determinan los comportamientos opuestos de los agelastoi (los que no ríen nunca) y de los gelastoi (los que ríen siempre) ${ }^{72}$. Joubert atribuye a la melancolía, al humor negro del que habla Aristóteles en el problema Treinta, el hecho de que ciertos filósofos no se han abandonado jamás al placer de la risa ${ }^{73}$. Al contrario, Gutberleth formula, a su manera, una hipótesis que vuelve a menudo en la literatura dedicada a este sujeto:

Entre los hombres serios que sobresalen en sabiduría y en prudencia, la risa es bastante rara a causa tanto de sus pensamientos, que se dirigen hacia cosas serias, como de su amplia experiencia, que tiene como consecuencia que les suceden pocas cosas insólitas e inusitadas ${ }^{74}$.

por A. Tassoni ( Perché ne dilettino le favole, ancorché sappiamo, che non contengano verità» (Quisito V), en Prose politiche e morali, cit., vol. II, p. 228).

${ }^{71}$ "On dit aussi de Socrate (tres renommé pour sa grande sagesse) qu'il etoit tousjours de maime visage, ne plus joyeux, ne plus troublé. Platon fut si modeste, qu'on ne le vit jamais rire, sinon moyennement»: L. Joubert, Traité du ris, cit., p. 250. Cf. también G. Cardano (De Subtilitate libri XXI, Basilea, Per Sebastianum Henricpetri, 1611, p. 649 ( $1^{\mathrm{a}}$ ed., Nuremberg, 1550) e I. P. Valeriano ( Risus», en Hieroglyphica, Basileae, por Thomam Guarinum, 1567, p. 44).

72 Acerca de agelastoi y gelasinoi: R. Maffei, De risu et fletu, Commentariorum urbanorum...octo et triginta libri, Basileae, in Officina Frobeniana, 1530, p. 382: I. L. Vives, De risu, en De anima et vita libri tres, Basileae, Per Ioannem Oporinum, s. d., p. 225; Fracastor, "De admiratione et Ecstasi et risu», en De sympathia et antipathia rerum, cit., p. 123; G. Ferrari, Democrito et Eraclito. Dialoghi del Riso, delle Lagrime e della Malinconia, cit., p. 4; E. Puteani (Eric Van de Putte), De risu, en Suada attica sive Orationum selectarum syntagma, Lovanii, Typis Io. Christoph. Flavii, 1615, pp. 375376. Rabelais emplea varias veces el vocablo «agélaste»: D. Ménager, La Renaissance et le Rire, cit., pp. 80-81.

73 L. Joubert, Traité du ris, cit., pp. 255-262. Acerca del problema XXX de Aristótes, enteramente dedicado a la relación entre genio y melancolía, $c f$. la introducción de J. Pigeaud a su Aristote. L’homme de génie et la mélancolie, París, 1988, p. 9-78.

74 «... in viris gravis sapientia et prudentia praecellentibus rarior est risus et pressior, quum ob profundas et ad res ferias intentas cogitationes, tum quod ob multam experientiam pauca illis occurrunt insueta et nova»: H. Gutberleth, "Laetitiae accidunt Gratulatio et Risus», en Pathologia hoc est Doctrica de Humanis affectibus, Herbornae Navassiorum, 1615, p. 26. Acerca de la razón por la cual los filósofos no rien, $c f$. también F. Valleriola, "De risus natura et causis et ad quam potissimum partem referri debeat: quidque ab eo consequatur» en Enarrationum medicinalium libri sex, Lugduni, Apud Sebastianum Gryphium, 1554, p. 215. 
Si la risa se vincula con el estupor y si el estupor es producto de la novedad, los sabios ríen raramente o no ríen nunca, porque las cosas desconocidas que se les pueden presentar son poco numerosas. Lo que es objeto de estupor para el hombre común no puede serlo para quien hace de la cogitatio («reflexión») una profesión cotidiana. Cornelio Ghirardelli es de opinión contraria. Sólo el hombre que ríe muestra aptitud para el conocimiento, porque «la risa es lo propio del hombre», con lo cual se quiere significar que el hombre es un animal racional y, por tanto, capaz de sentir placer en el acto de conocer:

Por lo tanto, quien no comprende algo es necesariamente triste y la inteligencia de quien es triste e incapaz de alegrarse es necesariamente tan cargada que le resulta imposible aprender las cosas de manera suficiente para disfrutar de ellas ${ }^{75}$.

Esta posición de risa moderada se opone a las otras dos posiciones extremas: la de los agelastoi que no ríen nunca y la de las mujeres, de los locos y de los niños que ríen sin parar. La ausencia de risa o su presencia excesiva son incompatibles con cualquier forma de conocimiento. No podemos dedicar más tiempo a estas cuestiones fascinantes y tal vez extrañas, pero debemos al menos añadir aquí una aclaración importante que vincula la teoría del relato a la teoría de la risa. En la definición de Bonciani, que hemos examinado al principio, se afirma que las novelle sieno imitazione d'una intera azione cattiva secondo il ridicolo («los relatos son la imitación de una completa acción mala según lo ridículo»). Con «acción mala» se debe entender lo que los comentaristas de Aristóteles del siglo XVI habían incluido en la fórmula turpitudo sine dolore: según el Estagirita, en efecto, lo ridículo procede de una turpitudo, de insuficiencias y defectos que no deben ser ni dolorosos ni mortales. En presencia de acontecimientos traumáticos o chocantes, será imposible echarse a reír:

La comedia es, como hemos visto, la imitación de hombres de calidad moral inferior, no en todas las especies de vicios, sino en el dominio de lo risible, que es una parte de lo feo. Porque lo risible es un defecto y una fealdad sin dolor ni daño. Así, por ejemplo, la máscara cómica es fea y deforme sin expresión de dolor ${ }^{76}$.

Situaciones de perversión o de maldad están excluidas del horizonte del relato, porque più tosto arrecano agli uomini dolore che allegrezza ("procuran

75 "Dunque chi non intende è forza che se ne stia mesto e chi sta mesto e non si sa rallegrare è forza c'habbia impedito di modo l'intelletto che non possa apprendere le cose, tanto che basti per farsi allegro», C. Ghirardelli, Cefalogia Fisonomica divisa in dieci Deche, Bolonia, Presso Clemente Ferroni, 1630, p. 159.

76 Aristóteles, Poética, 5, 1449 a 31-36. 
a los hombres más dolor que alegría» ${ }^{77}$. Esto por lo que concierne a Aristóteles. Sin embargo, cuando se trata de describir con atención las diferentes tipologías de turpitudo, recurriendo a unos exempla de Boccaccio o de Plauto, Bonciani, considerado un aristotélico de estricta observancia, combina las teorías de la Poética y las del Filebo de Platón. En la Lezione sopra il comporre delle novelle («Lección sobre la composición de los relatos»), en efecto, redacta una lista de nueve acciones ridículas que reelabora el esquema platónico. La risa deriva de la ignorancia de sí, de la distancia entre lo que uno se cree y lo que uno de verdad es, distancia evidenciada en la escena por el objeto cómico:

Y puesto que los relatos son imitación no sólo de una acción fea, sino de una acción fea según lo ridículo, no estará fuera de nuestros objetivos indicar brevemente cuál es el origen de dichas acciones, cuyo origen diremos que procede de la ignorancia que, a su vez, como dice Platón en su Filebo, depende de la estulticia. Ahora bien, esta ignorancia que se genera en nosotros por el hecho de razonar mal y siguiendo malos principios, nace de numerosas causas y nos afecta en diversas circunstancias. Por esto nos engañamos a propósito de los bienes o del ánimo o del cuerpo o de la fortuna y la razón de este nuestro engaño pueden ser o bien nuestro mal razonamiento, que nos lleva a engañarnos a nosotros mismos haciéndonos creer una cosa en lugar de otra, o bien la casualidad y la fortuna, que nos hacen caer en dicha ignorancia, o bien los hombres sagaces, que con su astucia nos brindan la ocasión para engañarnos ${ }^{78}$.

El que hace alarde de riquezas, de cualidades físicas y de virtudes que no posee provoca naturalmente la risa. La mezcla de temas platónicos y aristotélicos no es ciertamente un hecho nuevo y no concierne sólo a Bonciani. Trissino, Maggi, Castelvetro y Riccoboni también asocian a la turpitudo sine dolore unas reflexiones sacadas del Filebo o del Sofista ${ }^{79}$. Lo que sorprende, en cualquier caso, es la persistencia, en estos autores, del uso de etiquetas rígidas que nos despistan.

77 Francesco Bonciani, Lezione sopra il comporre delle novelle, cit., p. 112.

78 «E perché le novelle sono imitazione non solo d'una azione brutta, ma d'una tale secondo 'l ridicolo, non sarà fuor di proposito accennare così brievemente donde queste azioni traggano la loro origine, la quale diremo procedere dall'ignoranza che (si come piace a Platone nel Filebo) dalla stoltizia depende. Ora, per questa ignoranza che in inoi si genera per discorrer male e con cattivi principii, nasce da parecchie cause e circa varie e diverse cose ci occorre: e perciò che noi ci inganniamo intorno a' beni o dell'animo o del corpo o della fortuna; e la cagione di questo nostro inganno può essere o 'l nostro cattivo discorso, che noi da per noi stessi ci inganniamo dandoci ad intendere una cosa per un'altra, o vero il caso e la fortuna che per sorte ci fanno cadere in tale ignoranza, o pure gl'uomini sagaci i quali con la loro astuzia ci prestino occasione d'ingannarci»: ibid., p. 154.

79 Acerca de esta contaminación entre Platón y Aristóteles, $c f$. N. Ordine, Teoria della novella..., cit., pp. 76-82. 
En el debate relativo a las tipologías de lo feo, la dimensión erótica ocupa sin duda alguna un lugar significativo. Bonciani invita a «cubrir» metafóricamente las escenas más atrevidas mediante astucias lingüísticas y estrategias narrativas. El relato de un adulterio, por ejemplo, debe salvar el honor de las mujeres, atribuyendo la transgresión ocasional a la voluntad impredecible del azar. Aunque el relato cómico no deba jamás renunciar a la hipotiposis ${ }^{80}$ -a la habilidad de construir unas acciones que parezcan materializarse bajo los ojos del lector-, el relato de qualche cosa disonesta («de algo deshonesto») debe hacerse con onesti vocaboli («con palabras honestas») ${ }^{81}$.

De una manera más explícita, Castelvetro, en su comentario a la Poética de Aristóteles, clasifica el diletto carnale ( "placer carnal») entre las causas más importantes que provocan la risa. Mostrar las membra vergognose ( los miembros vergonzantes»), los congiungimenti lascivi («las uniones lascivas») o cosas parecidas suscita en el público una diversión asegurada, si uno respeta las reglas de la decencia. En cualquier caso, la escena debe ser cubierta por un velo. El autor debe saber mostrar y ocultar al mismo tiempo:

Por tanto, las cosas divertidas arriba mencionadas nos hacen reír cuando, en presencia de otras personas, se nos presentan tras unos velos, gracias a los cuales quedará claro que no nos estamos riendo de los que ellas tienen de deshonesto, sino de otras cosas, lo cual, sin necesidad de poner ejemplo alguno, es más que evidente para cualquiera. O bien, cuando se nos presentan sin velo, mediante hechos o palabras o de otra manera en un lugar en el que nadie nos ve $\mathrm{e}^{82}$.

El relato desnudo, sin velos, divertirá sólo en ausencia de público, cuando quien ríe no pone en peligro su propio honor. De esto es testimonio un relato del Novellino:

Érase una vez un hombre de natural tan grande que no encontraba a nadie que lo tuviera igual de grande. Ahora bien, un día sucedió que este hombre se encontró con una mujer que ya no estaba en la flor de su juventud y que, siendo muy fea, pero rica, había visto y catado a muchos. Cuando estuvieron en la habitación,

80 Bargagli subraya esta hipótesis: Dialogo, p. 224. Se trata de una figura importante en los tratados de retórica y particularmente en la Institutio oratoria (IV, 2, 123) de Quintilian.

81 Francesco Bonciani, Lezione sopra il comporre delle novelle, cit., p. 134.

82 «Adunque le cose predette piacentici ci fanno rider quando ci sono in presenza altrui presentate sotto alcuno velame, per mezzo del quale possiamo fare vista di non ridere della disonestà, ma d'altro; e ciò, senza darne esempio, è vie più che manifesto ad ognuno; o vero quando ci sono presentate senza velame in fatto o in parole o altramente in luogo che noi non siamo veduti da niuno": L. Castelvetro, Poetica d'Aristotele vulgarizzata e sposta, ed. W. Romani, Bari, 1978, p. 135. 
él se lo enseñó. La mujer, por la gran alegría, se echó a reír. El otro dijo: «¿Qué te parece?», y la mujer contestó $[\ldots]^{83}$

Joubert examina con atención el poder de la imagen obscena y el vínculo de ésta con la turpitudo, insistiendo, sobre todo, en su efecto-sorpresa, en su capacidad de dejar pasar valores claramente opuestos a la moral corriente:

Lo que vemos feo, deforme, deshonesto, indecente, insano y poco conveniente excita en nosotros la risa, con tal de que no nos mueva a compasión. Por ejemplo: si se descubren las partes vergonzantes, las que estamos acostumbrados a esconder por naturaleza o por honestidad pública, éstas, siendo feas, pero indignas de piedad, despiertan en quienes las ven la risa ${ }^{84}$.

Aun así, queda por aclarar un problema: el que concierne a las reacciones diversas del público ante una escena cómica. En efecto, no todo el mundo reacciona de la misma manera. Es por esta razón que los autores de tratados consideran legítimo preguntarse si la intensidad de un ataque de risa es determinada por la fuerza misma de la acción cómica o si, al contrario, es preciso atribuirla a la naturaleza particular de cada individuo. Maggi analiza cuidadosamente el problema. Si afirmamos, en efecto, que la risa depende de la naturaleza de los hombres, excluimos automáticamente que pueda existir una fuerza cómica inherente a lo ridículo; si, al contrario, pensamos que la risa es provocada exclusivamente por la vis comica inherente a lo ridículo mismo, todos deberíamos reír de la misma manera ante un acontecimiento cómico. Sin embargo, para Maggi, las dos hipótesis así formuladas no pueden solucionar la dificultad. Reconocer, en efecto, la fuerza autónoma de lo cómico no significa excluir la posibilidad que esta última se perciba de manera diferente según las diferentes naturalezas de cada individuo:

No hay que decir ni una cosa ni la otra. Esta dificultad, en nuestra opinión, puede suprimirse si decimos que lo que provoca la risa de la forma más eficaz reside en lo ridículo, en lo que aporta la causa de la risa. El hecho de que, aun así, no todos ríen de la misma manera ante la misma realidad ridícula no se debe a la realidad ridícula en sí, cuya eficacia es la misma en todos, sino a la propia naturaleza de los hombres, porque la naturaleza no nos constituye ni hace a todos del mismo modo ${ }^{85}$.

83 «Fu uno, ch'avea si grande naturale, che non trovava neuno che fosse si grande ad assai. Or avvenne che un giorno si trovà con una putta, che non era molto giovane; ed avegnaché molto fosse orrevole e ricca, molti n'avea veduto e provati. Quando furo in camera, ed elli lo mostrà. E per grande letizia, la donna rise. Quel disse: - Che ve ne pare? - E la donna rispose... »: Il Novellino, ed. a cargo de G. Manganelli, Milán, 1989³, p. 97.

84 L. Joubert, Traité du ris, cit., pp. 16-17.

85 «Sed neutrum horum dicendum. Hanc difficultatem tolli posse existimamus, si ad movendum risum maximam in ridiculo vim dixerimus esse, eo quod ridendi causam 
Joubert, médico cultivado, examina las diferentes complexiones humanas. Entre los dos polos extremos, el de los agelastoi y el de los gelasinoi, es posible individuar toda una serie de actitudes intermedias. En todo caso, los temperamentos y la cantidad de sangre juegan un papel considerable. Analizando estos dos elementos, será posible dibujar una tipología de diferentes actitudes, es decir, comprender por qué las mujeres y los niños ríen más que los hombres, los gordos más que los delgados, los hombres del pueblo más que los hombres de ciencias ${ }^{86}$. En estos análisis, pues, la risa se vuelve la expresión de la varietas («variedad») humana, así como el relato pone en escena los sucesos más diversos que conciernen a los hombres. De la misma manera, la naturaleza huidiza de la risa se vincula con la naturaleza huidiza del relato, con la naturaleza de un género literario difícil de clasificar debido a la fluidez de sus formas continuamente en relación con la de otros géneros como el diálogo, la comedia, la epístola ${ }^{87}$. Fluidez de formas que Bonciani pone en evidencia y que no parece demasiado distante de la inestabilidad denunciada por los teóricos de la risa:

Siendo la risa una de esas cosas que no tienen un ser permanente o, por decirlo de forma más clara, un ser estable, porque su ser reside en su hacerse continuamente, no es posible atribuir a la risa un verdadero género ni una verdadera diferencia $[\ldots]$ como se hace con las realidades permanentes y estables ${ }^{88}$.

Tal vez sea por estas razones que las consideraciones de Cicerón relativas a los inevitables efectos «cómicos» de la literatura dedicada a la risa parecen pesar como una amenaza no sólo sobre las elecciones de los teóricos del Renacimiento, sino también sobre las de todos aquellos que siguen midiéndose con este sujeto:

Viendo un día ciertas obras griegas dedicadas al humor, me dejé llevar por la esperanza de poder aprender algo. Encontré ciertamente en ellas un buen número de esas salsas picantes, tan comunes entre los griegos (porque los Sículos,

afferat; quod tamen omnes non aeque rideant eodem audito ridiculo, id evenit non ipsius ridiculi ratione, cuius vis eadem est apud omnes, sed ipsius hominum naturae, quoniam non omnes eodem modo sunt ac constituti ac a natura facti»: V. Maggi, De ridiculis, dans Trattati di poetica e retorica del Cinquecento, ed. a cargo de B. Weinberg, vol. II, Bari, 1970, p. 118.

86 L. Joubert, Traité du ris, cit., pp. 249-266.

87 Acerca de las interferencias entre el relato y los demás géneros literarios, $c f$. $\mathrm{N}$. Ordine, Teoria della novella..., cit., p. 91.

88 "Per essere il riso una di quelle cose che non hanno alcun essere permanente, o per dir più chiaramente stabile, ma che continuamente sta in farsi non se gli si può così facilmente attribuire alcuno vero genere, ne alcuna vera differenza [...] come si fa con le permanenti e stabili»: B. Parravicino, Discorso del riso vera proprietà dell'huomo, Como, 1615, p. 17. 
los Rodios, los Bizantinos y, sobre todos, los Áticos sobresalen en este género), pero los que han intentado dar una explicación del humor y deducir de ello una teoría han resultado ser tan insulsos que uno se ríe nada más que de su misma insulsez ${ }^{89}$.

89 "Itaque cum quosdam Graecos inscriptos libros esse vidissem de ridiculis, nonnullam in spem veneram posse me ex iis aliquid discere; inveni autem ridicula et salsa multa Graecorum; nam et Siculi in eo genere et Rhodii et Byzantii et praeter ceteros Attici excellunt; sex qui eius rationem quandam conati sunt artemque tradere, sic insulsu existerunt, ut nihil aliud eorum nisi ipsa insulsitas rideatur»: Cicerón, De Oratore, II, 217. 


\section{RECENSIONES}


